\title{
MÖBIUS CONE STRUCTURES ON 3-DIMENSIONAL MANIFOLDS
}

\author{
FEng LuO
}

\begin{abstract}
A вsтract. We show that for any given angle $\alpha \in(0,2 \pi)$, any closed 3manifold has a Möbius cone structure with cone angle $\alpha$.
\end{abstract}

In dimension three, there are eight complete locally homogeneous geometries. Five of them $\left(S^{3}, H^{3}, E^{3}, H^{2} \times R^{1}\right.$, and $\left.S^{2} \times R^{1}\right)$ are naturally conformally flat. W. Goldman in [Go] showed that manifolds modeled on Sol or Nil geometry cannot have conformally flat structures. As to the last geometry $\widetilde{\mathrm{PSL}}(2, R)$, Gromov-Lawson-Thurston [GLT], Kapovich [Ka], and Kuiper $[\mathrm{Ku}]$ showed that some of the closed 3-manifolds modeled on $\widetilde{\mathrm{PSL}}(2, R)$ geometry have conformally flat structures.

In this note, we announce that,

Theorem 1. For any positive $r<2$ any closed 3-manifold $M$ has a Riemannian metric ds (singular) of the following form. There are local coordinates $(z, t)$ ( $z$ complex and $t$ real) in $M$ so that in the coordinates the metric ds is:

(a) conformally flat: $d s=u(z, t)\left(|d z|^{2}+|d t|^{2}\right)$ or,

(b) conformally flat with cone singularity of angle $r \pi$,

$$
d s=u(z, t)\left(|d z|^{2} /|z|^{2-r}+|d t|^{2}\right)
$$

where $u(z, t)$ is a smooth positive function of $z, t$.

Furthermore, if $r=2 / n$ for some positive integer $n>1$, then the monodromy group is a discrete subgroup of $S O(4,1)$.

We may also state the result in terms of Möbius cone geometry as follows. Given $\alpha \in(0,2 \pi)$, an $\alpha$-cone 3 -sphere $S_{\alpha}^{3}$ is the quotient of a Euclidean lens of angle $\alpha$ by a rotation about the edge of the lens which identifies the two boundary half-spheres of the lens. The above result is the same as:

1991 Mathematics Subject Classification. Primary 57M50, secondary 57M99.

Received December 7, 1993. 
Theorem 2. Given any positive $\alpha<2 \pi$, any closed 3-manifold $M$ has a singular conformally flat structure so that each point in $M$ has a neighborhood which is conformal to an open set in $S_{\alpha}^{3}$. Furthermore, if the given cone angle is $2 \pi / n$ for some $n \in Z_{+}$, then the monodromy group is a discrete subgroup of $S O(4,1)$.

The singularity forms a link in the manifold. We call the singular conformal structure a Möbius cone structure with cone angle $\alpha$.

The technical details of the proof will appear elsewhere. We will describe below the basic idea of the proof. Essentially, we show that 3-dimensional Dehn surgery can be realized in Möbius cone geometry. The basic idea of the proof comes from Gromov-Lawson-Thurston's construction in [GLT].

Recall that three-dimensional Möbius geometry $\left(S^{3}, \operatorname{Mob}\left(S^{3}\right)\right)$ has underlying space the unit 3-sphere or, equivalently, Euclidean 3-space together with infinity, $\bar{R}^{3}=R \bigcup\{\infty\}$. The Möbius transformations are the same as those homeomorphisms which are compositions of inversions about 2spheres. Circles and spheres are invariant under Möbius transformations. Given a circle in $S^{3}$, the half-turn about the circle is the orientation preserving Möbius involution leaving the circle pointwise fixed.

The following notions introduced by Kuiper $[\mathrm{Ku}]$ are crucial to our construction. A necklace in $R^{3}$ is a union of finitely many closed balls $B_{1}, B_{2}, \ldots, B_{n}$ in $R^{3}$ so that only adjacent index balls $B_{i}$ and $B_{i+1}$ intersect transversely (the index is counted $\bmod (n)$ ). The complement of the interior of a necklace in $S^{3}$ is called a Möbius $n$-gon $P$. The intersection of $P$ with a closed ball $B_{i}$ is called a side of $P$ and the intersection $\partial B_{i} \cap \partial B_{i+1}$ is called an edge of $P$. A Möbius annulus is a topological annulus in a sphere bounded by two disjoint circles. The middle circle of a Möbius annulus is the circle in it so that the inversion about the circle leaves the annulus invariant. An orthogonal arc in a Möbius annulus is a circular arc orthogonal to the two boundary components.

For simplicity, we will describe the idea of the proof of Theorem 2 in the case that $\alpha=\pi$ and the manifold is orientable.

It is known from the work of Lickorish [Li] that any closed orientable 3 -manifold is obtained by doing \pm 1 -Dehn surgeries on the complement of a closed pure braid in $S^{3}$. Our goal is to realize this surgery construction in Möbius cone geometry.

We first cover each component of the braid by small balls so that their union forms a necklace with small exterior angles. These necklaces are all disjoint and form a regular neighborhood of the braid. The edges of the Möbius polygon are the meridian curves of the braids. We will start a sequence of modification in each necklace to achieve the Dehn surgery. Suppose $N$ is such a necklace with cyclically ordered sides $A_{1}, A_{2}, \ldots, A_{n}$, 
and suppose $H_{i}$ is the half-turn about the middle circle of $A_{i}$ for each $i$. We then introduce an identification on $\partial N$ by these half-turns, i.e., each side $A_{i}$ is self-identified by $H_{i}$. The quotient space will be homeomorphic to the \pm 1 -Dehn surgeries on the braid if we choose the necklace suitably. To see this, take the edge $E=A_{1} \cap A_{n}$ of the Möbius $n$-gon $\operatorname{int}(N)^{c}$. The Möbius transformation $\phi=H_{n} \circ H_{n-1} \circ \cdots \circ H_{1}$ sends $E$ to itself. Thus, points $x, \phi(x), \phi^{2}(x), \ldots$ in $E$ are all identified in the quotient. Thus the quotient is a manifold if and only if $\phi$ is periodic, i.e., $\phi^{k}=$ id in $E$ for some integer $k$. We require that $k=1$ :

(1) $\phi=$ id in $E$.

Since all edges are identified to one edge, we also need

(2) the sum of the exterior angles of $N$ is $2 \pi$.

Assume that (1) holds. Then the quotient is homeomorphic to an integer coefficient Dehn surgery on $\partial N$. To see this, take $t \in E$, let $t_{i}=H_{i} \circ \cdots \circ$ $H_{1}(t)$, and let $c_{i}$ be the orthogonal arc in the Möbius annulus $A_{i}$ with end points $t_{i}$ and $t_{i+1}$. Then the simple closed curve $C=\bigcup_{i} c_{i}$ in $\partial N$ is invariant under the identification and $C$ intersects a meridian of $N$ at one point. The identification in $\partial N$ together with $C$ can be described as follows. Let $P$ be a Euclidean $n$-sided polygon in the plane, let $m_{1}, \ldots, m_{n}$ be the middle points of the sides of $P$, and let $h_{i}$ be the Euclidean rotation of angle $\pi$ about $m_{i}$. Then the quotient of $P$ by the identification on $\partial \mathrm{P}$ induced by $h_{i}$ on the $i$-th side containing $m_{i}$ is a 2 -sphere. The quotient of $P \times S^{1}$ under the identifications $h_{i} \times i d$ on the $\{i$-th side $\} \times S^{1}$ in $\partial P \times S^{1}$ is $S^{2} \times S^{1}$. The identification on $\partial N$ is the same as the one on $\partial P \times S^{1}$ and the invariant curve $C$ is the same as $\partial P \times\{p\}$. This shows that the quotient of $\operatorname{int}(N)^{c}$ is homeomorphic to the Dehn surgery on $\partial N$ killing $C$. The coefficient of the Dehn surgery is the linking number between $C$ and the core of the necklace $N$. We call the linking number the twist number of a Möbius polygon satisfying (1).

(3) The twist number of the Möbius polygon is \pm 1 (depending on the coefficient of the Dehn surgery).

Having recognized the topology of the quotient, we now modify the necklace to achieve (1), (2), (3). We first construct a necklace $N$ so that (1) holds and the sum of the exterior angles of $N$ is $\pi$. Suppose the twist number of $\operatorname{int}(N)^{c}$ is $k$. To achieve (3), we will glue a regular Möbius necklace to $N$ along one side. That condition (3) can be realized is morally supported by the Gromov-Lawson-Thurston's construction [GLT] of regular Möbius polygon. We prove that there is a regular Möbius n-gon so that (1) holds; (2) the sum of its inner angles is $\pi$; and (3) the twist number is $k \pm 1$ (any given integer). Having constructed this regular Möbius $m$-gon, we now modify $N$ so that (1) still holds, the sum of inner angles is again $\pi$, 
the twist number is still $k$, and one side $S$ of $\mathrm{N}$ has the same module as the sides of the regular Möbius m-gon. By attaching this regular polygon to $N$ along the side $S$, we find a necklace satisfying all the required conditions. Thus the result follows. Note that the group generated by half-turns on all of the middle circles is a discrete group by the Poincaré polyhedron theorem.

Note that if we use the periodic condition $\phi^{k}=$ id in $E$ in condition (1), then it corresponds to the rational coefficient Dehn-surgery.

To modify the cone angle to any arbitrary positive number $\alpha<2 \pi$, we replace the Möbius annulus $A_{i}$ by the Möbius lens which is a union of two Möbius annuli $A, B$ along one of their boundary components $L$ at an angle $2 \pi-\alpha$ so that the degree $\alpha$ rotation about $L$ takes $A$ to $B$.

It seems highly possible due to the solution of the Yamabe problem by Schoen $[\mathrm{Sc}]$ that there is a constant scalar curvature metric of the same form (a) and (b) in the conformal class of $d s$, and the metric is unique if the singular set is non-empty and the manifold pair ( $M$, singular set) is not $\left(S^{3}\right.$, circle). We are informed by $\mathrm{G}$. Tian that such a constant scalar curvature metric exists if the integration of the scalar curvature of a given singular metric in the conformal class is non-positive.

One would hope that under some topological assumptions on $M^{3}$ these Möbius cone structures have a limit as the cone angle tends to $2 \pi$. This would lead to the existence of conformally flat structures on $M^{3}$. Unfortunately, the topological condition is that $\pi_{1}\left(M^{3}\right)$ is trivial. Indeed, any reasonable convergence of cone structures would imply the algebraic convergence of the monodromy groups. Since the monodromy group of $M^{3}-S$ ( $S$ denotes the singular set) is trivial and $\pi_{1}\left(M^{3}-S\right.$ ) is mapped surjectively to $\pi_{1}\left(M^{3}\right)$ under the inclusion map, thus $\pi_{1}\left(M^{3}\right)=1$ is a necessary condition for the existence of the limit. On the other hand, one may start with a Dehn surgery description of the given 3-manifold $M^{3}$ in any conformally flat manifold $N^{3}$. The above construction still works in $N^{3}$. This produces cone structures on $M^{3}$ with non-trivial monodromy groups on $M^{3}-S$.

\section{Acknowledgement}

Part of this work was done at the University of California at San Diego when the author was visiting there. He thanks Professor Michael Freedman for discussions and encouragement.

\section{References}

[Go] Goldman, W., Conformally flat manifolds with nilpotent holonomy, Trans. AMS. 278 (1983), 573-583. 
[GLT] Gromov, M., Lawson, B., Thurston, W., Hyperbolic 4-manifolds and conformally flat 3-manifolds, Math. Publ. IHES. 68 (1988), 27-45.

[Ka1] Kapovich, M., Conformally flat structures on 3-manifolds, J. Diff. Geom. 38 (1993), 191-215.

[Ku] Kuiper, N., Hyperbolic 4-manifolds and tesselations, Math. Publ. IHES. 68 (1987), 47-76.

[Li] Lickorish, W., A representation of oriented combinatorial 3-manifolds, Ann. Math. 72 (1962), 531-540.

[L1] Luo, F., Constructing conformally flat structures on some 3-manifolds, Math. Ann. 294 (1992), 449-458.

[L2] Luo, F., Möbius cone structures on 3-manifolds, in preparation.

[Sc] Schoen, R., Conformal deformation of a Riemannian metric to constant scalar curvature, J. Diff. Geom. 20 (1984), 233-238.

Department of Mathematics, Rutgers Univerity, New Brunshick, NJ 08903

E-mail address: fluo@math.rutgers.edu 